\title{
Nano biosensors for neurochemical monitoring
}

\author{
M. Meyyappan
}

\begin{abstract}
Neurochemicals such as dopamine (DA) and serotonin (S-HT) are linked to disorders such as Parkinson's disease, epilepsy, addiction and many others. Detection of and monitoring these neurochemicals in vivo and in vitro has become important in treating various disorders. The electroactive nature of DA and S-HT has enabled employing electrochemical techniques to detect them at low concentrations, and a variety of electrodes and approaches have been reported. The use of nanomaterials such as carbon nanotubes, graphene and nanowires has been advocated in recent years for the sensitive detection of neurochemicals. This article reviews the advances in nano biosensors for this application and discusses the future outlook and challenges.
\end{abstract}

Keywords: Biosensors; Nanomaterials; Neurochemical detection; Carbon nanotubes; Graphene; Neural disorders

\section{Introduction}

Research activities on nanomaterials such as carbon nanotubes (CNTs), graphene, various inorganic nanowires and nanoparticles have grown exponentially over the last two decades. The nanomaterials offer interesting and unique electrical, mechanical, optical and other properties compared to their bulk counterparts. Exploiting these differences to construct useful devices, systems and architecture is expected to benefit all economic sectors, and the research activities span all areas of science and engineering [1-4]. Sensor technology is one of the areas to reap the benefits of nanoscience and technology, offering tremendous potential to develop sensors for early warning of infectious diseases, cancer diagnostics, and lab-on-a-chip for rapid and inexpensive routine health monitoring involving blood and urine analysis [5]. A subset of medical diagnostics to benefit from nanosensors includes sensitive detection of neurochemicals for the treatment of various neural disorders such as Parkinson's disease, epilepsy, etc. [6-8]. This article first provides a brief background on neurochemicals, biosensors and nanomaterials and then reviews the sensor development efforts to date. This is followed by a detailed discussion of carbon nanofiber electrodes developed in our laboratory for this purpose and finally, future outlook and challenges are summarized.

\subsection{Neurochemicals and the need for monitoring}

Neurotransmitters are chemicals in the brain that transmit signals from the neurons to the target cells. Dopamine

Correspondence: m.meyyappan@nasa.gov

NASA Ames Research Center, Moffett Field, CA 94035, USA
(DA) and Serotonin (5-HT) are two of these chemicals, which have been associated with a number of neural disorders [9-15]. Dopamine concentration is associated with Parkinson's disease, epilepsy and addiction. Insufficiency of dopamine is thought to cause Parkinson's disease whereas excess release of DA leads to schizophrenia. Similarly, serotonin levels are linked to disorders such as addiction and depression. Both DA and HT-5 are present in very small quantities in a sea of ascorbic acid (AA) with $100-1000$ times higher concentration. Most of the detection techniques used in the literature and reviewed here, especially the electrochemical techniques, have the advantage of fast response time, but the real challenge is the selectivity between DA and HT-5 in AA. The two neurochemicals have oxidation potentials close to each other, separated only by $150 \mathrm{mV}$. Therefore, many conventional electrodes have difficulty yielding very distinct peaks for the two targets especially when both are present together in AA at realistic concentration levels. This has prompted the investigation of nanomaterial based electrodes or modification of conventional electrodes with coatings of nanomaterials.

In addition to recording the concentrations of neurochemicals, electrodes are desired for stimulation as well $[9,10]$ since some of the neural disorders are treated by deep brain stimulation (DBS). The stimulation is believed to produce evoked release of the neurochemicals. Therefore, monitoring the released chemicals adds to the importance of developing sensitive techniques to provide feedback for the DBS procedure and improve 
the outcome. Figure 1 shows a schematic of the electrode inserted in the brain for DBS, which is typically $1.3 \mathrm{~mm}$ in diameter with four $1.5 \mathrm{~mm}$ contacts. This electrode is used for stimulation only at present without any feedback from monitoring the concentrations of neurochemicals. Targeted activation of specific and precise location with a much smaller electrode along with guidance/ feedback from measured neurotransmitter levels is highly desirable. Nanoelectrodes have the potential to meet this challenge, and the requirements would include ultrahigh sensitivity $\sim 1 \mathrm{nM}$, fast speed of $10 \mathrm{~ms}$ resolution and reliability and stability for long term implantation.

\subsection{Biosensors}

Biosensors are devices that provide the surface to host the probe-target interaction and translate this interaction into some form of a measurable signal. The signal output can be in many forms: optical, deflection of a cantilever, electrical or electrochemical signals $[5,16-$ 19]. The International Union of Pure and Applied Chemistry (IUPAC) defines a biosensor as "a device that uses specific biochemical reactions mediated by isolated enzymes, immunosystems, tissues, organelles, or whole cell to detect chemical compounds usually by electrical, thermal or optical signals" [5]. Of these, optical methods have been well developed and shown capable of detecting down to a single molecule. They are commonly fluorescence based wherein the bio-recognition molecules or probes are labeled with fluorescent tags (example: dye) and the probe-target interaction results in a fluorescent signal. The intensity relates to the concentration of the target molecules and capture efficiency. Though labeling is laborious, label-free optical techniques have been developed as well.

Electrical biosensors may take the form of bio field effect transistor (BioFET) wherein the conventional transistor gate is replaced with a liquid gate and a reference electrode $[18,19]$. When a probe is attached to the gate, the current-voltage (I-V) characteristics would shift from the behavior of the unmodified gate. Further probe-target interaction would bring about additional shift of the I-V curve proportional to the concentration of the target molecules in the solution. BioFETs have been extensively investigated for the detection of various biomarkers related to healthcare and environmental monitoring.

Another form of electrical transduction involves electrochemical techniques that use metal (Pt for example) or carbon electrodes. The latter can be in the form of graphite, glassy carbon, carbon paste or diamond, which have long been in use; more recently, carbon nanotubes and graphene have become popular as well $[1,3,5]$. These electrodes are typically used in amperometric, potentiometric or impedimetric modes wherein the electrodes are functionalized with probes that have selectivity or affinity for the targets of interest $[16,17]$. The probes in all cases can be DNA, RNA, antibodies, aptamers etc.

\subsection{Nanomaterials}

Since their discovery in the early 1990s, carbon nanotubes have commanded significant attention in terms of

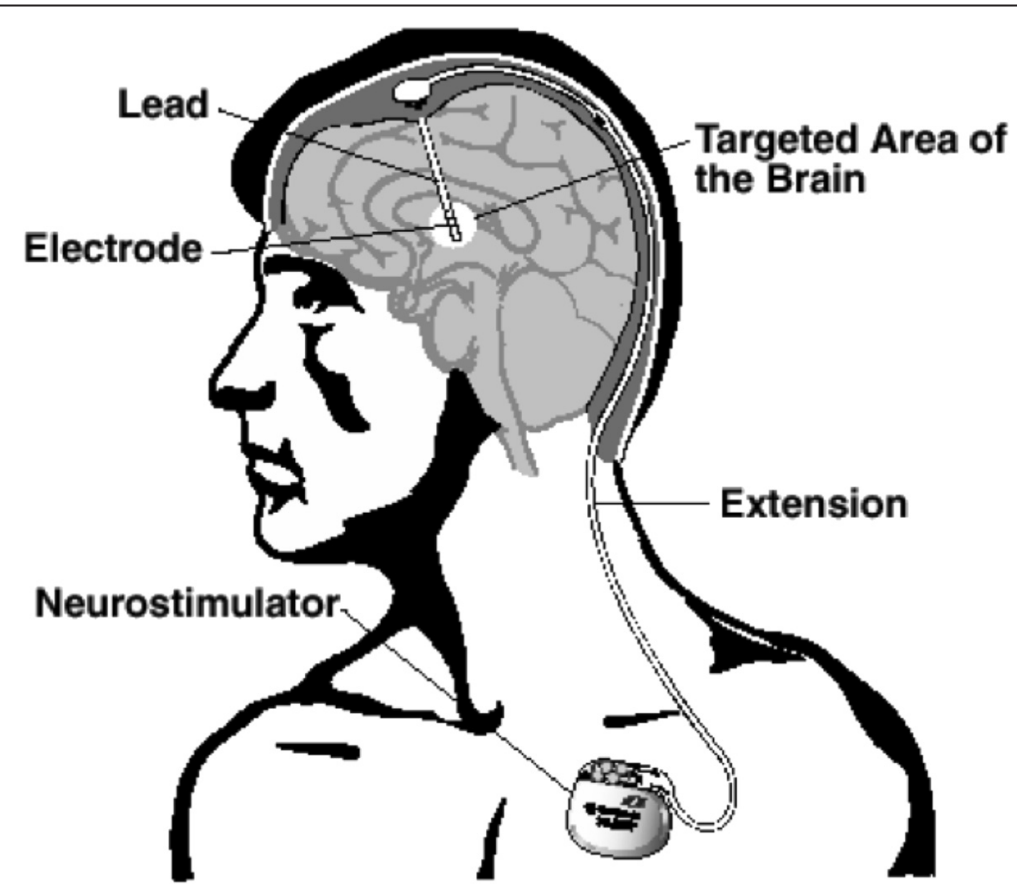

Fig. 1 Schematic of the electrode in deep brain stimulation. Image courtesy of Jun Li 
growth, characterization of their interesting properties and application development [1]. The CNTs are seamless cylinders arising from the roll-up of graphene sheets. In the case of single-walled carbon nanotubes (SWCNTs), a chiral vector $(n, m)$ defines their intrinsic properties. When $(n-m) / 3$ is an integer, the resulting structure is metallic; otherwise, it is semiconducting. Multiwalled carbon nanotubes (MWCNTs) are concentric cylinders with a hollow core, resembling rolled-up graphite sheets. Both SWCNTs and MWCNTs are grown by techniques such as arc synthesis, laser ablation, chemical vapor deposition (CVD) and plasma enhanced chemical vapor deposition (PECVD). While all of these approaches can produce CNTs in bulk quantities, CVD and PECVD are suited to grow CNTs on substrates with controlled thickness and even vertical orientation, suitable for device fabrication. The properties of CNTs can be modified by functionalizing them with other atomic or molecular groups. For example, fluorination makes SWCNTs insulating [20]. Attaching bio probes of DNA, RNA, aptamers, antibodies etc. enables development of biosensors for selective capturing of biotargets of interest $[1,5]$. CNTs have been investigated for a wide variety of applications including transistors, memory devices, chem, bio and radiation sensors, nanoelectromechanical devices, high strength light weight composites, batteries, supercapacitors, filters and membranes, thermal interface materials, electrical interconnects in integrated circuits, electrical cables, transparent electrodes, shielding of electromagnetic interference, field emission devices, catalyst support and many others [1].

Graphene is a one atom thick quasi two-dimensional material made up of $\mathrm{sp}^{2}$-bonded carbon atoms, which are packed in a honeycomb-like lattice. It is a semimetal, that is, a zero-gap semiconductor. Graphene exhibits a very low resistivity of $\sim 10^{-6} \mathrm{ohm}-\mathrm{cm}$ and a high mobility of $\sim 15000 \mathrm{~cm}^{2} / \mathrm{v} . \mathrm{s}$ [3]. The Young's modulus of graphene is estimated to be $\sim 1 \mathrm{TPa}$. Graphene is synthesized by exfoliation of graphite or reduced graphite oxide. CVD also is a popular method to produce graphene films on nickel and copper substrates. PECVD can provide multilayer graphene vertically oriented to the substrate surface. Graphene has been investigated for almost every application listed above for CNTs.

Thin films of various inorganic materials such as $\mathrm{Si}$, Ge, GaAs, InP, oxides, nitrides etc. have helped with advances in microelectronics, optoelectronics, microelectromechanical systems and others. In recent years, most of these materials have been grown in the form of onedimensional (1D) nanowires. When the radius of a semiconducting nanowire is smaller than its Bohr radius, the bandgap increases from the corresponding value for the thin film. Nanowires offer interesting electrical, optical, thermal and other properties relative to their bulk counterparts and therefore, have received much attention in the development of electronic, photonic, sensor and other devices [2].

\section{Review}

\subsection{Sensors for neurochemical monitoring}

All of the nanomaterials mentioned above have been used for the detection of neurochemicals [21-59]. A review of selected works is presented here. Baldrich et al. [21] prepared SWCNT electrodes through entrapment of nanotubes on the surface of protein-coated magnetic particles. This electrode was able to distinguish the peaks of DA and uric acid (UA) at $200 \mu \mathrm{M}$ concentrations. Li et al. [22] modified glassy carbon electrodes (GCEs) with SWCNTs to obtain a DA detection limit of $50 \mathrm{nM}$ with peak current linearly varying between 5 and $100 \mu \mathrm{M}$. A graphite electrode modified with MWCNTs and pretreated for DA detection enabled Moreno et al. [23] to obtain a linear response in the range of $50 \mathrm{~nm}-1 \mu \mathrm{M}$ in the presence of AA. Electrodes using a nanocomposite of MWCNTs, sol gel silica and gold nanoparticles were tried by Komathi et al. [24] for the sensitive detection of DA. The sensitivity was found to be nearly four times smaller without the gold nanoparticles. The pores in the silica network host MWCNTs which are hydrophobic and silica with $-\mathrm{NH}_{2}$ group which are hydrophilic. The latter attract the ascorbate ions and reject the DA molecules, which reside on the surface of MWCNTs and exhibit strong electron transfer reactions. In addition, the presence of gold nanoparticles enhances the electrocatalytic oxidation of DA. The linear range for DA detection reported in this work is 0.1 to $30 \mathrm{nM}$. Yang et al. [25] prepared a composite of nano-sized copper oxide / MWCNTs / nafion and used it to modify a GCE which showed a detection limit of $0.4 \mu \mathrm{M}$. Aravind and Ramaprabhu [26] created a nanocomposite of Pt nanoparticles and MWCNTs and immobilized it with SS-DNA to prevent nanotube agglomeration. The SS-DNA also facilitated electron transfer reaction with dopamine. This hybrid electrode was shown to have a DA detection limit of $0.8 \mu \mathrm{M}$. Kumaraswamy and Venton [27] modified a carbon fiber microelectrode with SWCNTs and used it for the co-detection of DA and ST in vivo in the striatum of anesthetized rat after administration of a serotonin synthetic precursor. The measured concentrations $24 \mathrm{~min}$. after the administration were $250 \mathrm{nM}$ for $\mathrm{DA}$ and $130 \mathrm{nM}$ for seratonin.

Kim et al. [28] modified a glassy carbon electrode (GCE) with graphene to reduce the capacitive background current and increase the current intensity of DA. This allowed them to obtain a complete peak separation between DA and AA with a linear range for DA detection between 4 and $100 \mu \mathrm{M}$. Alwarappan et al. [29] also used a graphene-modified GCE but reported selective detection results only for $1 \mathrm{mM}$ each of DA, AA and serotonin. Sun et al. [30] modified a glassy carbon electrode with graphene/Pt to obtain distinct peaks in DPV curves for AA, 


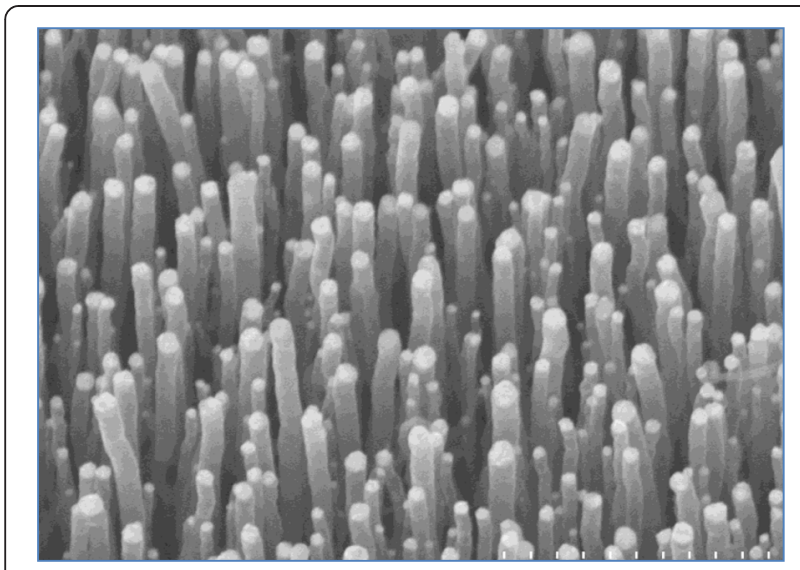

Fig. 2 Vertical carbon nanofibers grown by plasma enhanced chemical vapor deposition. The nanofibers have a diameter of $\sim 50 \mathrm{~nm}$ and height of 3-4 $\mu \mathrm{m}$. Image courtesy of Prabhu Arumugam
DA and UA. The $1.7 \mathrm{~nm}$ Pt particles self-assembled on graphene showed better performance than GC and $\mathrm{GC} /$ graphene electrodes. Modification of GCE with a nanocomposite of $\beta$-cyclodextrin and graphene sheet enabled Tan et al. [31] to obtain a linear current response in the range of $9 \mathrm{nM}$ to $12.7 \mu \mathrm{M}$ DA in PBS. The linear range obtained in the amperometric mode was from 0.9 to $200 \mu \mathrm{M}$. In both cases, the nanocomposite-modified GCE did better than both GC and GC/graphene electrodes; this was attributed to the mass transfer control of the electrochemical reaction of dopamine on the nanocomposite in contrast to the usual adsorption-limited process. Han et al. [32] modified a GCE with a graphene-chitosan composite and compared it against a GC-chitosan electrode for the detection of DA, AA and UA. The addition of graphene was shown to increase the electrocatalytic activity towards the oxidation of all three chemicals. Gao et al. [33] modified a GCE with graphene oxide and showed a detection limit of $0.27 \mu \mathrm{M}$ DA in the presence of AA and the oxidation peak current showed a linear relation with
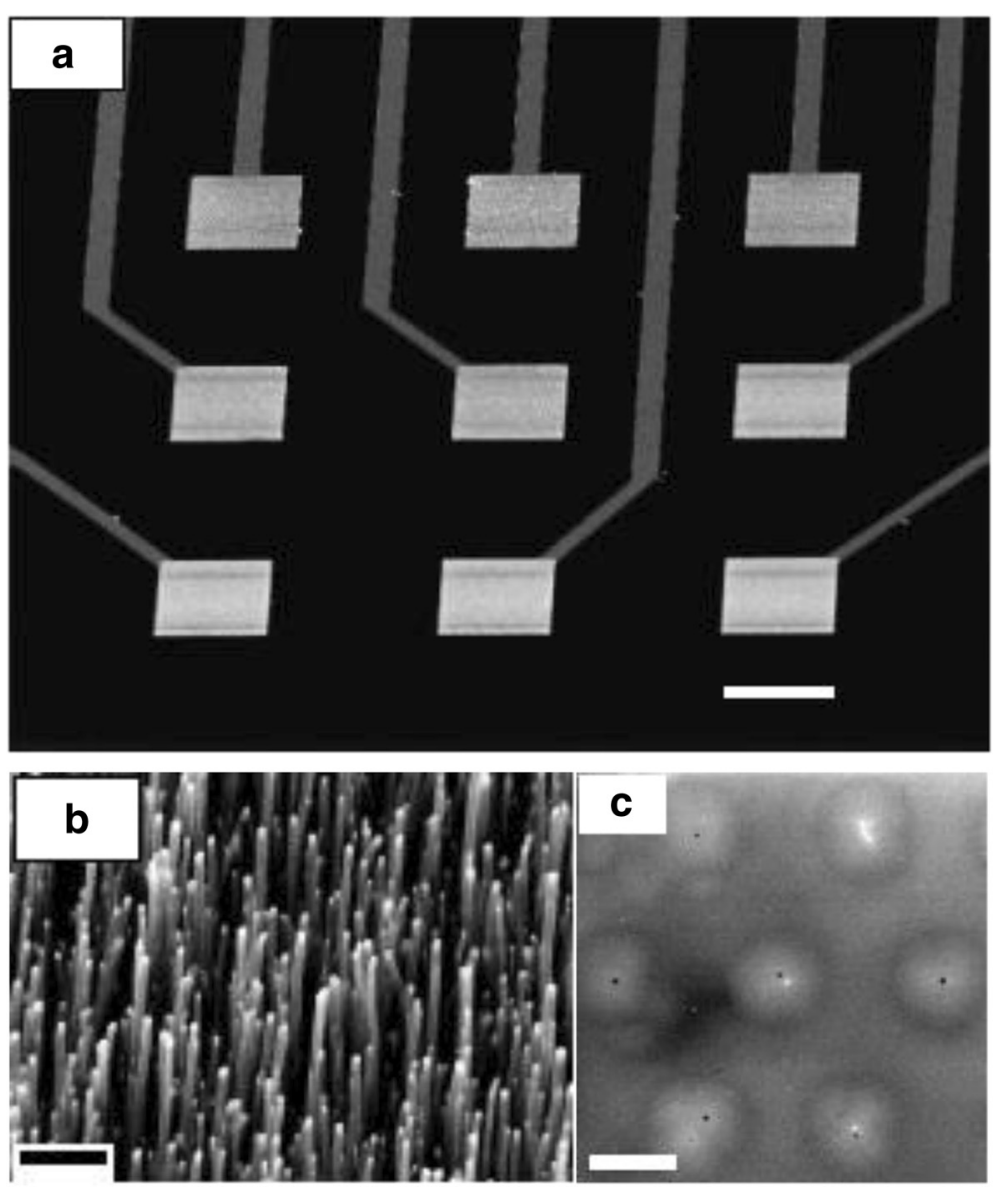

Fig. 3 (a) A $3 \times 3$ electrode array, (b) stimulating electrode consisting of CNFs with large area (scale bar: $1 \mu \mathrm{m})$ and (c) recording electrode embedded in $\mathrm{SiO}_{2}$ with high sensitivity (scale bar: $1 \mu \mathrm{m}$ ). Image courtesy of Jun Li 


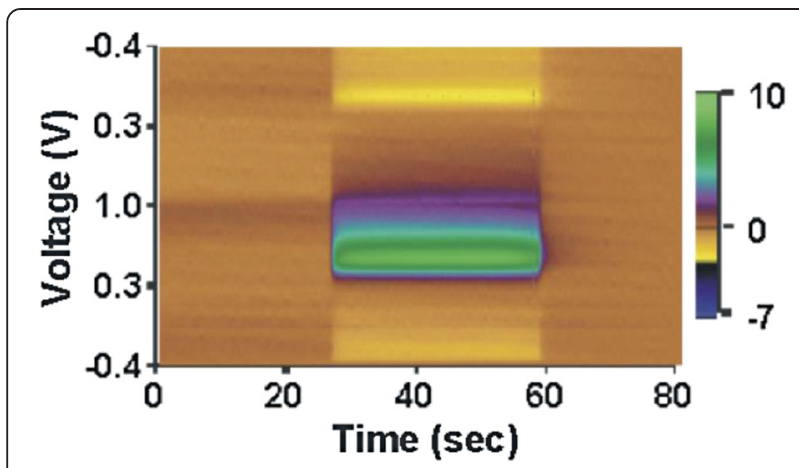

Fig. 4 Electrode response to injected dopamine $(2.5 \mu \mathrm{M})$ in real time [56]. Image courtesy of Jessica Koehne

concentration in the range of 1.0-15.0 $\mu \mathrm{M}$. Thomas et al. [34] modified a carbon paste electrode with graphene oxide to obtain a DA detection limit of $15 \mathrm{nM}$ in the presence of 1000-fold concentration of AA and UA. Sun et al. [35] prepared a nanocomposite of graphene and tin oxide nanosheets and used it to modify a carbon ionic liquid electrode. This modified electrode provided peak currents proportional to DA concentration in the range of 0.5 to $500 \mu \mathrm{M}$ with a detection limit of $0.13 \mu \mathrm{M}$. Tsai et al. [36] coated GCEs with Te nanowires and the modified electrode was further coated with nafion to improve the stability and selectivity.

Chandrashekar et al. [38] electropolymerized L-arginine on a carbon paste electrode to form a biopolymer and utilized it for the detection of DA, AA and UA. The peak current in the $\mathrm{CV}$ measurement showed a linear range for dopamine concentration between $50 \mu \mathrm{M}$ and $0.1 \mathrm{mM}$ with a detection limit of $0.5 \mu \mathrm{M}$. In addition to CNTs and graphene, diamond based electrodes have also been explored. For example, Raina et al. [39] used a nitrogenincorporated nano-diamond based ultramicroelectrode array and produced steady state CV curves for DA concentrations in the range of $100-800 \mu \mathrm{M}$ in $0.1 \mathrm{M}$ PBS.

Besides electrochemical approaches such as the ones discussed above, BioFETs have been used as well for detecting dopamine. For example, Li et al. [40] fabricated an open gate ion sensitive field effect transistor and measured the I-V characteristics for increasing DA concentrations from $1 \mathrm{fM}$ to 1 $\mathrm{nM}$. However, no data was given on how the device would perform when DA is present in mixtures of AA and/or UA.

\subsection{Carbon nanofiber electrodes for neurochemical monitoring}

Our group has been developing carbon nanofiber based nanoelectrodes for the detection of neurochemicals and also for stimulation in collaboration with Mayo Clinic [56-59]. The CNFs are $\sim 50 \mathrm{~nm}$ in diameter, grown by plasma enhanced chemical vapor deposition (PECVD). The as-grown structures are vertical and free-standing, as shown in Fig. 2, due to the electric field perpendicular to the wafer. The broken walls of CNFs along the outside of the structure as evidenced by TEM analysis (data not shown here) and the resulting defects are ideal for good electron transfer. The fabrication of the electrodes has been described previously [60-64] and a brief description is provided below with the fabricated electrodes in Fig. 3.

A silicon wafer with a $500 \mathrm{~nm}$ thick thermal oxide is used as substrate and a thin layer of $\mathrm{Pt}$ or $\mathrm{Cr}$ is deposited on the substrate to provide electrical contact between the CNFs and the measurement circuit. This is followed by sputtering of $10-30 \mathrm{~nm}$ nickel as catalyst layer. Acetylene is used to grow CNFs at a pressure of 1-3 Torr and temperature of 600$700{ }^{\circ} \mathrm{C}$. The catalyst film breaks up into tiny droplets of random sizes on the wafer and growth on these catalysts particles yields nanofibers shown in Fig. 3 (b). These uncoated CNFs can be used as stimulating electrode of desirable size, even down to a single CNF electrode of $50 \mathrm{~nm}$. Patterning of the catalyst instead of a blanket sputtered catalyst layer can provide CNFs of specified diameter at desired locations. The gap between the nanofibers is filled with $\mathrm{SiO}_{2}$ to provide mechanical stability. Then the top surface of the wafer with CNFs is subjected to chemical mechanical polishing which results in a smooth oxide surface with few nm of CNFs protruding out as seen in Fig. 3 (c). This embedded electrode is suitable for recording neurochemical concentrations.

Figure 4 shows a color plot of the response of the electrode shown in Fig. 3 (c) to injected dopamine $(2.5 \mu \mathrm{M})$ in
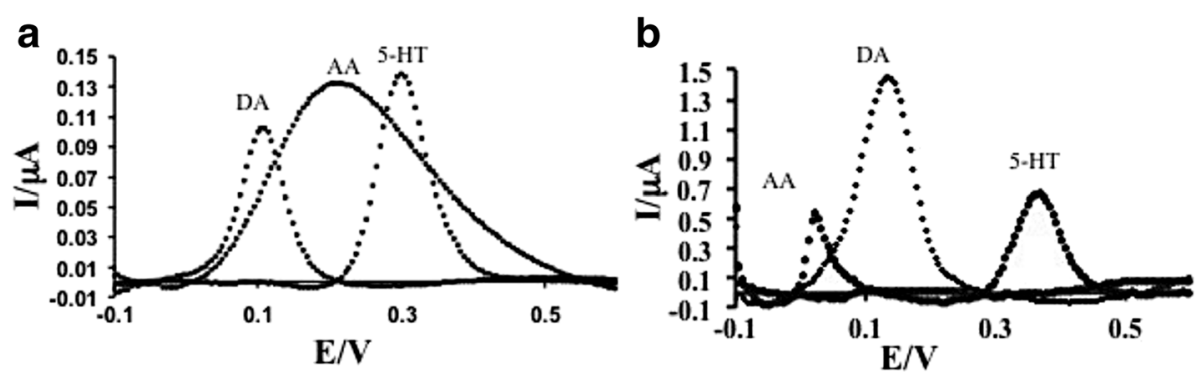

Fig. 5 Differential pulse voltammetry results for $1 \mathrm{mM} \mathrm{AA}, 10 \mu \mathrm{M}$ DA and $10 \mu \mathrm{M}$ 5-HT [58]. (a) glassy carbon electrode and (b) carbon nanofiber electrode. Image courtesy of Jessica Koehne 
real time. The response is seen $25 \mathrm{~s}$ after injection, ending around $60 \mathrm{~s}$ [56]. Fig. 5 compares the performance of this electrode with that of a conventional GCE for a mixture containing $1 \mathrm{mM}$ of $\mathrm{AA}$ and $10 \mu \mathrm{M}$ each of DA and 5-HT. The GCE is not able to resolve the peaks whereas the CNF nanoelectrodes shows very distinct peaks in the differential pulse voltammetry plots [58].

\section{Conclusions}

A review of the current status of sensors for the detection and monitoring of neurochemicals has been presented. Most electrodes do not have any difficulty in detecting down to few $\mathrm{nM}$ concentrations of dopamine by itself in buffer solutions. However, sensitive detection of DA in mixtures of 5-HT, ascorbic acid and others is challenging. Nanoelectrodes using CNTs, graphene and CNFs have been reported to meet these challenges at present. More studies demonstrating the utility of these techniques in vivo are needed. Nanoelectrodes for stimulation show much potential but no significant demonstrations have appeared yet. This is an area that requires further investigation.

\section{Competing interests}

The author declare that he has no competing interests.

\section{Acknowledgements}

The author acknowledges Jessica Koehne, the Biosensor Group Leader at NASA Ames Center for Nanotechnology who is responsible for Technology development and other past and present members of this team Russell Andrews, Jun Li, Prabhu Arumugam, Adai Periyakaruppan, Emily Rand, Ramprasad Ghandiraman and collaborators from Mayo Clinic Kendall Lee, Mike Marsh and Kevin Bennett.

Received: 17 March 2015 Accepted: 6 May 2015

Published online: 08 September 2015

\section{References}

1. M Meyyappan (ed.), Carbon Nanotubes: Science and Applications (Boca Raton, FL, CRC Press, 2004)

2. M Meyyappan, M Sunkara, Inorganic Nanowires: Applications, Properties and Characterization, (Boca Raton, FL, CRC Press, 2010)

3. W B Choi, J W Lee (eds.), Graphene Synthesis and Application (Boca Raton, FL CRC Press, 2012)

4. C Altavilla, E Ciliberto (eds.), Inorganic Nanoparticles Synthesis, Applications and Perspectives (Boca Raton, FL, CRC Press, 2011)

5. J Li, N Wu (eds.), Biosensors Based on Nanomaterials and Nanodevices (Boca Raton, FL, CRC Press, 2014)

6. RJ Andrews, Neuromodulation: Deep Brain Stimulation, Sensory Neuroprostheses and the Neural-Electrical Interface, in Progress in Brain Research, ed. by HS Sharma, 2009. 180, 127

7. RJ Andrews, Nanotechniques for Neurosurgery, Chapter 28, in Current Progress in Neurosurgery, Vol 1, ed. by BK Misra, AH Kaye, ER Laws (Kothari Medical Publishers, Mumbai, 2014), pp. 454-474

8. RR Llinas, KD Walton, M Nakao, I Hunter, PA Anquetil, J. Nanopart. Res. 7, 111 (2005)

9. F Agnesi, SJ Tye, JM Bledsoe, CJ Griessenuer, CJ Kimble, GC Sieck, KE Bennett, PA Garris, CD Blaha, KH Lee, J. Neurosurg. 111, 701 (2009)

10. KH Lee, SY Chang, DW Roberts, U Kim, J. Neurosurg. 101, 511 (2004)

11. S Kapur, G Remington, Am. J. Psychiatry 153, 466 (1996)

12. Y Ni, EB Malarkey, V Parpura, J. Neurochem. 103, 1273 (2007)

13. RM Wightman, JM Leslie, AC Michael, Anal. Chem. 60, 769A (1988)

14. DL Robinson, A Hermans, AT Seipel, RM Wightman, Chem. Rev. 108, 2554 (2008)

15. TJ Webster, MC Waid, JL McKenzie, RL Price, JU Ejiofor, Nanotechnol. 48, 48 (2004)

16. JS Daniels, N Pourmond, Electroanal. 19, 1239 (2007)
17. J Gooding, Electroanal. 14, 1149 (2002)

18. T Rim, KY Kim, CH Baek, YH Jeong, JS Lee, M Meyyappan, J. Nanosci. Nanotechnol. 14, 273 (2014)

19. F G Banica, Chemical Sensors and Biosensors, (Wiley, New York, 2012)

20. ET Michelson, CB Huffman, AG Rinzler, RE Smalley, RH Hauge, JL Margrave Chem. Phys. Lett. 296, 188 (1998)

21. E Baddrich, R Gomez, G Gabriel, IX Munoz, Biosens. Bioelec. 5, 1876 (2011)

22. Y Li, J Du, J Yang, D Liu, X Lu, Colloid Surf. B. Bioint. 97, 32 (2012)

23. M Moreno, AS Arribas, E Berijo, M Chicharro, A Zapardiel, MC Rodriguez, Y Jalit, GA Rivas, Talanta 80, 2149 (2010)

24. S Komathi, Al Gopalan, KP Lee, Analyst 135, 397 (2010)

25. S Yang, G Li, Y Yin, R Yang, J Li, L Qu, J. Electroanal. Chem. 703, 45 (2013)

26. SSJ Aravind, S Ramaprabhu, Sens. Actuat. B. Chem. 155, 679 (2011)

27. BE Kumaraswamy, BJ Venton, Analyst 132, 876 (2007)

28. YR Kim, SY Bong, YJ Kang, YT Yang, RK Mahajan, JS Kim, H Kim, Biosens. Bioelec. 25, 2366 (2010)

29. S Alwarappan, A Erdem, C Liu, CZ Li, J. Phys. Chem. C 113, 8853 (2009)

30. CL Sun, HH Lee, JM Yang, CC Wu, Biosens. Bioelec. 26, 3450 (2011)

31. L Tan, KG Zhou, YH Zhang, HX Wang, XD Wang, YF Guo, HL Zhang, Electrochem. Commun. 12, 557 (2010)

32. D Han, T Han, C Shan, A Ivaska, L Niu, Electroanal. 22, 2001 (2010)

33. F Gao, X Cai, X Wang, C Cao, S Liu, F Gao, QX Wang, Sens. Actuat. B. Chem. $186,380(2013)$

34. T Thomas, RJ Mascarenhas, C Nethravathi, M Rajamathi, BE Kumaraswamy, J. Electroanal. Chem. 659, 113 (2011)

35. W Sun, X Wang, Y Wang, X Ju, L Xu, G Li, Z Sun, Electrochem. Acta. 87, 317 (2013)

36. HY Tsai, ZH Lin, HT Chang, Biosens. Bioelec. 35, 479 (2012)

37. S Minnikanti, MGAG Pereira, S Jaraiedi, K Jackson, CM Costa-Neto, Q Li, N Peixoto, J. Neural Eng. 7, 016002 (2010)

38. BN Chandrashekar, BE Kumaraswamy, M Pandurangachar, TV Sathisha, BS Sherigara, Colloid Surf. B. Bioint. 88, 413 (2011)

39. S Raina, WP Kang, JL Davidson, Diamond Rel. Mater. 19, 256 (2010)

40. DC Li, PH Yang, MSC Lu, IEEE Trans. Electron Dev. 57, 2761 (2010)

41. MPN Bui, CA Li, GH Seong, Biochip. J. 6, 149 (2012)

42. MS Lu, YC Chen, PC Huang, Biosens. Bioelec. 26, 1093 (2010)

43. S Sansuk, E Bitziou, MB Joseph, JA Corington, MG Boutelle, PR Unwin, JV MacPherson, Anal. Chem. 85, 163 (2013)

44. S Yang, G Li, R Yang, M Xia, L Qu, J. Solid State Electrochem. 15, 1909 (2011)

45. M Zhu, C Zeng, J Ye, Electroanal. 23, 907 (2011)

46. TV Sathisha, BE Kumaraswamy, S Reddy, BN Chandrashekar, B Eswarappa, J. Mol. Liq. 172, 53 (2012)

47. X Yu, K Sheng, G Shi, Analyst 139, 4525 (2014)

48. H Kim, JH Lee, EJ Chae, JW Choi, BK Oh, J. Nanosci. Nanotech. 11, 7516 (2011)

49. D Sun, Q Zhao, F Tans, X Wang, J Gao, Anal. Methods. 4, 3253 (2012)

50. JH Jin, E Cho, SH Jung, Biotechnol. Lett. 32, 413 (2010)

51. AN Patel, SY Tan, TS Miller, JV MacPherson, PR Unwin, Anal. Chem. 85, 11755 (2013)

52. M Hasanzadeh, X Shadjou, E Omidinia, J. Neurosci. Methods 219, 53 (2013)

53. P Veerakumar, R Madhu, SM Chen, CT Hung, PH Tang, CB Wang, SB Liu, Analyst 139, 4994 (2014)

54. TDB Nguyen-Vu, H Chen, AM Cassell, R Andrews, M Meyyappan, J Li, Small 2, 89 (2006)

55. TDB Nguyen-Vu, H Chen, AM Cassell, RJ Andrews, M Meyyappan, J Li, IEEE Trans. Biomed. Eng. 54, 1121 (2007)

56. JE Koehne, M Marsh, A Boakye, B Douglas, IY Kim, SY Chang, DP Jang, KW Bennet, C Kimble, R Andrews, M Meyyappan, KH Lee, Analyst 136, 1802 (2011)

57. MP Marsh, JE Koehne, RJ Andrews, M Meyyappan, KE Bennett, KH Lee, Biomed. Eng. Lett. 2, 271 (2012)

58. E Rand, A Periyakaruppan, Z Tanaka, DA Zhang, MP Marsh, RJ Andrews, KH Lee, M Meyyappan, JE Koehne, Biosens. Bioelect. 42, 434 (2013)

59. DA Zhang, E Rand, M Marsh, RJ Andrews, KH Lee, M Meyyappan, J Koehne, Mol. Neurobiol. 48, 380 (2013)

60. JE Koehne, H Chen, AM Cassell, Q Ye, J Han, M Meyyappan, J Li, Clinical Chem. 50, 1886 (2004)

61. PU Arumugam, H Chen, S Siddiqui, JAP Weinrich, A Jejelowo, J Li, M Meyyappan, Biosens. Bioelect. 24, 2818 (2009)

62. PU Arumugam, E Yu, R Riverie, M Meyyappan, Chem. Phys. Lett. 499, 241 (2010)

63. A Periyakaruppan, RP Gandhiraman, M Meyyappan, JE Koehne, Anal. Chem. 85, 3858 (2013)

64. RK Gupta, A Periyakaruppan, JE Koehne, M Meyyappan, Biosens. Bioelect. 59, $112(2014)$ 